\title{
EDUCATION AND TRAINING The role of extended reality technology in healthcare education: Towards a learner-centred approach
}

The use of extended reality (XR) technologies is growing rapidly in a range of industries from gaming to aviation. However, how this technology should be implemented in healthcare education is not well-documented in the literature. Learner-driven implementation of educational technology has previously been shown to be more effective than a technology-driven approach. In this paper we conduct a narrative literature review of relevant papers to explore the role of XR technologies in learner-driven approaches to healthcare educatio. This paper aims to evaluate the position of XR technologies in learner-centred pedagogical models, determine what functions of XR technologies can improve learner-centred approaches in healthcare education, and explore whether XR technologies can improve learning outcomes in healthcare education. We conclude that XR technologies have unique attributes that can improve learning outcomes when compared to traditional learning methods, but there is currently a shortfall in learner-centred implementation of XR technologies in healthcare education, where these technologies have the capacity to cause a paradigm shift.

KEYWORDS: health education and training, extended reality, immersive technology, virtual reality, augmented reality

\section{DOI: 10.7861/fhj.2020-0112}

\section{Introduction}

Extended reality (XR) encapsulates a range of tools that blend physical and virtual environments, ${ }^{1,2}$ the three major subdivisions being virtual, augmented and mixed reality (Box 1). Over the last decade there has been a drive to introduce these technologies into healthcare education, motivated by a number of potential benefits

Authors: ${ }^{A}$ Topol digital health fellow, Health Education England; ${ }^{B}$ senior clinical advisor, Health Education England; 'ST6 ophthalmology, University Hospitals Birmingham NHS Trust; Phead of technology enhanced learning, Health Education England; Eknowledge specialist, Health Education England

\section{Box 1. Categories of extended reality technologies ${ }^{3-5}$}

Extended reality (XR) is an overarching term that encapsulates current and future developments in augmented reality, mixed reality and virtual reality.

Augmented reality (AR): The superimposition of digital data onto the real world

Mixed reality (MR): A seamless integration of both virtual and digital environments that may be interacted with

Virtual reality (VR): A technology that possesses three main characteristics:

$>$ Primarily first (sometimes third) person perspectives within the scenario

Real-time interaction with a virtual environment

The user is completely surrounded by the virtual environment

including scalability, enhanced motivation and cost savings. ${ }^{2,6-8}$ However, the introduction of this technology into healthcare education has not always delivered the expected benefits. The challenge is to ensure that the potential advantages conferred would result in enhanced learning outcomes.

There have been two main approaches to the implementation of $\mathrm{XR}$ in education: technology-driven and learner-driven.

Technology-driven approaches use the capabilities of the technology as the starting point for the development of learning materials. In contrast, learner-driven approaches use the learning process as the starting point. Several comparative assessments of technology and learner-driven approaches have been conducted, finding that technology-centred methods have underperformed in terms of returns from capital investment and non-superior learning outcomes. ${ }^{9}$ Learner-driven approaches focus on pedagogical frameworks, and how technology can be used to enhance them. Pedagogy refers to the 'interactions between teachers, students, and the learning environment and learning task. ${ }^{10}$ The value of following pedagogical theory lies in its ability to improve academic achievement, social development and acquisition of technical skills. ${ }^{11,12}$

Technology-driven approaches aim to use technology to deliver a learning experience. Learning driven approaches focus on the learning objective, and retrospectively review the best method of 
achieving it. Rather than asking the question - How can I use the $X R$ to deliver anatomy teaching, learning driven approaches ask what is the best method of learning anatomy?

\section{An overview of learning pedagogy}

The first major type of pedagogy is teacher-centred. The focus of this methodology is the teacher. More traditional methods such as didactic lectures, deliberate practice and rote memorisation fit within this realm. While aspects of this pedagogy such as deliberate practice have been found to be effective, critics of this approach highlight the risk of teacher dependency, limited knowledge mastery and poor cognitive development.11,13-15

The second major type of pedagogy is learner-centred, in which the student is the centre of the learning process and the teacher's role is primarily that of a facilitator. In this capacity the teacher tries to create structures and conditions that enhance the learning process for students. Learner-centred approaches have been shown to have high student/teacher satisfaction, better motivation and enhanced critical and problem thinking skills. ${ }^{11,13-15}$

The learner-centred approach encapsulates theories from Piaget's constructivism and Siemens connectivism. ${ }^{16,17}$ According to the theory of constructivism, when new information is learnt, it is evaluated against our pre-existing knowledge base and subsequently added to it. ${ }^{18}$ This is achieved through three main methods: experiential learning, collaborative learning, and situated learning/cognition.

Piaget and Dewey put forward the view that learning is dependent on exploration and experience with the surrounding environment (experiential learning). It is believed we are evolutionarily hard-wired to create an internal mental model of a complex world around us, often referred to as a cognitive schema. Thus, everyone's internal framework of reality will be unique. ${ }^{18,19}$ This evolutionary, constructivist approach to learning was extended further by Vygotsky. He proposed that we are inherently social beings, thus our interactions with other humans (collaborative learning) will influence our learning. ${ }^{20}$ Finally, Wilson and Meyers stress the importance of situated cognition, which states that all knowledge is acquired in a specific context. ${ }^{21}$

While both teacher-centred and learner-centred methods have their merits, healthcare education is primarily directed at undergraduates, postgraduates and working professionals. These educational settings require the use of higher cognitive skills and self-directed learning, and thus the focus of this paper will be on learner-centred theory. ${ }^{22,23}$

\section{XR and pedagogical frameworks}

To date, there is limited evidence of the role of XR within these pedagogical frameworks, and how they can be effectively utilised in healthcare education. An integrative review of augmented reality studies in healthcare education found that only $20 \%$ were guided by some form of learning theory. ${ }^{24}$ This is consistent with data from the wider education sector-where only $32 \%$ of technology has been developed on some form of learning pedagogy. ${ }^{25}$

The aims of this paper are as follows:

$>$ To evaluate the position of XR in learner-centred pedagogical models.

> To determine what functions of XR can improve learner-centred approaches in healthcare education.

$>$ To determine if XR can improve learning outcomes in healthcare education.
Box 2. Search strategy used in literature review

1. (immersive AND technolog*).ti,ab

2. (digital AND technolog*).ti,ab

3. (digitech OR 'digital technolog*').ti,ab

4. ('virtual reality' OR VR).ti,ab

5. ('augmented reality' OR AR).ti,ab

6. 1 OR 2 OR 3 or 4 or 5

7. ('medical education' OR 'health* education').ti,ab

8. 6 AND 7

9. (digital AND service*).ti,ab

10. 6 OR 9

11. (assess ${ }^{*}$ OR evaluat ${ }^{*}$ OR criteria OR rating OR score OR scoring OR framework).ti,ab

12. 10 AND 11

13. (teach $^{*}$ OR educat ${ }^{*}$ OR pedagog $\left.{ }^{*}\right)$.ti,ab

14. (method* OR strategy* OR tool* OR format* ${ }^{*}$ OR intervention).ti,ab 15. 13 AND 14

\section{Method}

We chose to conduct a narrative review according to Scale for the Assessment of Narrative Review Articles (SANRA) principles due to its broad scope, ubiquity and ability to include a range of information sources. ${ }^{26,27}$ While systematic reviews remain the gold standard in answering a specific question, narrative reviews have proven to be effective in answering broader research topics. ${ }^{27,28}$ The following databases were searched by two authors: PubMed, Medline, and the Health Management Information Consortium (HMIC) database. The search strategy used is given in Box 2. All studies that were included had a clear research question, appropriate study design and discussion of their results. Absence of any of these resulted in exclusion from the study. Additional articles that were relevant were hand-searched from Google Scholar, and also identified from citations within articles.

\section{Pedagogical evidence for the use of XR}

\section{Experiential learning}

Experiential learning asserts that knowledge acquisition is enhanced in environments where the learner can explore, experience and assimilate new information in the context of what they already know. XR has the unique advantage of giving users the freedom to explore knowledge and environments through means not usually afforded to them by traditional methods. This includes the ability to view situations through egocentric and exocentric vantage points. When the user is present in a virtual environment it enables them to view a problem either from within the situation (egocentric) or from the outside (exocentric). Egocentric views assist in knowledge acquisition, while exocentric views improve lateral thinking. ${ }^{29} \mathrm{XR}$ has a variety of input tools and multimodal feedback mechanisms (visual, haptic etc) that enable the user to manipulate their environment and respond accordingly. If any domains of knowledge conflict with an individual's cognitive schema, they have the opportunity to manipulate the environment to answer any of these internal questions. ${ }^{19,30,31}$

\section{Collaborative learning}

Vygotsky's model of social constructivism supports the notion that learning in a social context will enhance cooperation, idea 
exchange and enhance knowledge acquisition. XR can provide the foundation for this collaborative learning process, as multiple learners can adopt avatars and interact with each other in a virtual world. Collaborative learning in this fashion improves learner adaptability and critical thinking skills.20,30,32,33

\section{Situated cognition}

Immersive technologies provide a unique opportunity to take on the form of avatars with different characteristics and personalities. Accurate representations can create a strong sense of connection between the user and the avatar. This promotes free expression, creativity and imagination. Avatars that represent real-life professionals will support situated cognition, and potentially better knowledge transfer to the real world. This is particularly helpful in the context of problem-based learning (PBL). XR enables realistic representations of scenarios, and learners can be assigned different roles to solve a problem. XR also enables the user to customise the scenario parameters and difficultly level, as well as providing immediate feedback..$^{30,33-36}$

\section{XR and connectivism}

A newer application of learner-centred pedagogy is connectivism. Siemens argues that while traditional pedagogical theories such as constructivism focus on the learning process, they place no value on what is being learnt. In an era of information abundance and technology, he argues we must question what knowledge is worth learning. Tenets of connectivism include the following:

> Learning requires the connection of multiple sources of information.

> Accurate and up-to-date knowledge is fundamental to all activities.

> Learners must have the ability to determine what to learn and form connections between a variety of ideas and concepts. ${ }^{17}$

The major manifestation of this pedagogy in the real world has been the form of massive open online courses (MOOCs)..$^{17}$ These are networks of people and resources that can be used to direct one's own learning. MOOCs run on the principle of lifelong education, democratisation of knowledge and generation of learning networks. Teachers typically play no role or are present in as a facilitator. A variety of tools exist for interacting with the including blogs, online meetings and virtual reality platforms.
However, the learning process has often been associated with poor interactivity, immersion and low participation levels. Zhang et al argues that XR offers the perfect opportunity to overcome these hurdles and make the learning process more enjoyable. They enable constructivist principles to be implemented within the realm of connectivism..$^{17,37}$ High levels of immersion support exploration and learning. There is the capacity to create virtual rooms and avatars to promote experiential and collaborative learning. XR can improve participation by offering enhanced student-teacher and student-student interaction with real-time feedback. ${ }^{17,37}$ The concept of immersion will be discussed in more detail later on.

\section{How does XR improve learning in healthcare education?}

In the previous section we explored the pedagogical basis for the use of XR technologies in education. We will now look at the unique capabilities of XR to enhance learning in the context of healthcare education. Fundamental to this are the interactions and responses that occur between the user and the technology. Several frameworks exist to evaluate this, but the StimuliOrganism-Response (SOR) framework proposed by Suh et al considers the antecedents, internal processes and consequences that arise from the use of XR technologies.

There are three components to the SOR framework: ${ }^{2}$

> Stimuli: any modality that initiates a cognitive or affective response within the user.

> Organism: the internal evaluation undertaken by the user.

> Response: outcomes from the use of immersive technology.

A summarised version of the SOR framework can be seen in Fig $1 .{ }^{2}$

Stimuli

Interactivity (perceptual stimuli)

This refers to the ability of the system to detect a user's input and respond. XR technology enables users to interact with learning material through a variety of unique modalities such as tactile inputs. ${ }^{2}$ A meta-analysis by Kyaw et al looked at the role of virtual reality in medical education, and showed that high levels of interactivity were associated with greater knowledge and skills acquisition. ${ }^{38}$ This covered a variety of domains including anatomy, ultrasound scanning and surgical simulators.

\section{Sensory stimuli Perceptual stimuli Content stimuli}

\section{Stimulus}

\section{Organism}

Cognitive reaction

Affective reaction

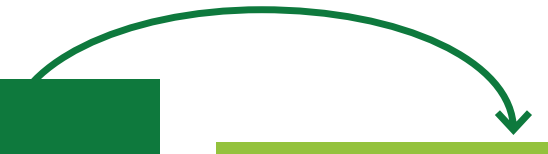

Positive outcomes

Negative outcomes

Response

Fig 1. Summary of the Stimuli-Organism-Response (SOR) framework produced by Suh and Prophet. ${ }^{2}$ 


\section{Box 3. Definitions}

Interaction fidelity: 'The objective degree of exactness with which real-world interactions can be reproduced. ${ }^{2} 3$ Important components include haptic feedback, controllers and response to voice

Display fidelity: 'The objective degree of exactness with which real-world sensory stimuli are reproduced. ${ }^{43}$ Important components include auditory output, visual output, stereopsis, field of view, resolution, and frames-per-second

Field of view (FOV): 'The relative field of the user's total view within which the environment's visuals extend' 44

Tracking level: 'The number and types of degrees of freedom (DOF) with which a user is tracked by an immersive system' 44 Frames-per-second: 'The number of times an image on the screen is refreshed each second' 3

Stereopsis: 'Recreating the experience humans get from seeing the real world with two eyes'3

\section{Representational fidelity (perceptual stimuli)}

This refers to the degree of realism. ${ }^{2}$ It has been a long-held theory that higher degrees of fidelity are beneficial for the learning process. ${ }^{39}$ However, a review by Norman et al did not show a significant difference in performance between high and low fidelity simulators. ${ }^{40}$ The primary focus of the study was on auscultation, basic motor and critical skills. This may limit the external validity of these findings. ${ }^{40}$ Alternatively, results from other studies show a distinct advantage from the use of highfidelity simulators. ${ }^{41,42}$ The current literature would suggest that a degree of fidelity is required that reflects the task complexity and the learner's ability. ${ }^{42}$ Other components of fidelity have not been covered in this paper, but the major types can be seen in Box 3 .

\section{Organism}

\section{Immersion/presence (cognitive reaction)}

Immersion refers to the ability of a system's sensorial information to reproduce reality and enable user engagement. Presence is a subjective aspect of the system that creates a perceptual illusion that the user is really there. ${ }^{45}$ For the purposes of this paper, we will not differentiate the two. Immersion is often regarded as the most significant advantage of XR when compared to traditional learning methods. ${ }^{46}$ The data on the role of presence appear to be conflicting: high levels of immersion have been shown to improve learning outcomes, ${ }^{47,48}$ but very high levels have also been shown to have negative consequences on knowledge acquisition. 30.49 The reason for this is not fully understood, but it is commonly attributed to the cognitive theory of multimedia. This states there are three processing demands placed on the user:

> essential (creation of simplified internal representations of the material being taught)

$>$ generative (the process of understanding the material driven by motivation)

$>$ extraneous (processing that does support the instructional objective)..$^{50}$

Immersion or presence has the capacity to increase both generative and extraneous processing. Immersion appears to be beneficial to a degree, but the design of a system must focus on learning objectives and avoid unnecessary distractions. 2,47,49,51 Distraction is not necessarily a detriment to learning. Many real-world environments have inherent distractions, and these can be emulated using XR, providing this is the desired learning objective.

A major meta-analysis by Cummings et al found that the most important contributors to presence were tracking level, stereopsis and field of view. ${ }^{44}$ Latency was associated with reduced presence and fidelity. Given the system burden that higher tracking levels and fields of view may confer, a balance must be found between this and excessive latency. ${ }^{51}$

\section{Response}

\section{Positive outcomes (learning effectiveness)}

It is important to validate XR software to see if it is producing the desired learning outcomes. Validity is 'the process of determining the degree to which a model is an accurate representation of the real world from the perspective of its intended uses. ${ }^{52}$ There are many methods of classifying validation. We will focus on two main branches of validity: those that focus on the appropriateness of the content (content-related) and those that focus on how the measures relate to others (criterion-related).

Content-related validity can be further subdivided into face, content and construct validity. Face and content validity are a basic measure that asks novice users and experts respectively to determine if the $\mathrm{XR}$ experience appears to measure what is purports to measure. This is a subjective measure that is typically captured using a Likert scale. Construct validity determines whether the XR experience is measuring what it claims to measure. ${ }^{53-55}$

Criterion-related validity is subdivided into concurrent and predictive validity. Concurrent validity compares the XR experience to another established measure (ie the gold standard) while predictive validity determines whether it can predict a criterion in the future. Ideally, all five domains should be present. ${ }^{53-55}$ Validation is not necessarily applicable across all XR devices and is dependent on the software rather than the hardware.

\section{Current validation of $X R$ in healthcare education} Barsom et al conducted a major systematic review that looked at the effectiveness of augmented reality in medical training, primarily on the basis of validation data. ${ }^{56}$ Seven major augmented reality applications were identified, but they failed to satisfy all five domains of validity. The most common forms of validation that were used were face and construct validity, which was demonstrated for a variety of tools such as echocardiography. ${ }^{56}$ There is a wide range of research into the use of virtual surgical training. Results show high levels of face, content and construct validity in a variety of surgical disciplines including laparoscopic and orthopedic procedures. ${ }^{57}$

Kyaw et al conducted a large meta-analysis on virtual reality in healthcare education that focused on concurrent validity. ${ }^{38}$ It compared the effectiveness of virtual reality to traditional learning methods (2D images, lectures etc). They also performed a subgroup analysis comparing the efficacy of knowledge vs skills acquisition. The study showed that virtual reality conferred better knowledge (standardised mean difference $[S M D]=0.44$; $95 \%$ confidence interval [CI] 0.18-0.69; $\mathrm{I}^{2}=49 \%$ ) and skills acquisition, although this was more apparent when looking at skills development $\left(\mathrm{SMD}=1.12 ; 95 \% \mathrm{CI} 0.81-1.43 ; \mathrm{I}^{2}=0 \%\right){ }^{38}$ 
Negative outcomes

The use of immersive technology does have some negative responses that could be detrimental to learning effectiveness. Motion sickness is a common complaint that appears to be more prevalent among women than men. It has also been shown that head-mounted displays confer a degree of postural stress that could have negative long-term consequences. In addition, variable degrees of eye strain have also been shown with the use of such technologies. ${ }^{2,58,59}$ As the technology and associated hardware becomes more widespread, it is conceivable that a new range of untoward effects will be identified.

\section{Discussion}

XR technologies such as virtual, augmented and mixed realities have the potential to enhance the quality and delivery of healthcare education. They could provide high-volume crosssite interactive learning at a lower cost. However, this must not come at the expense of high-quality educational outcomes. It is desirable that XR technologies offer non-inferior if not superior outcomes to traditional learning methods. The temptation might be to implement XR without a clear understanding of learning environments and objectives. Technology-driven instead of learnerdriven approaches often fail to provide sustainable improvements in learning outcomes and the learning environment. ${ }^{9}$ Other drivers for XR technologies in healthcare education should also be evaluated including cost effectiveness and accessibility. As a minimum, cost-benefit and cost-utility non-inferiority compared to other learning modalities should be present.

We believe that the value of XR is best demonstrated by the adoption of a learner-driven approach, in particular by applying learner-centred principles based on sound pedagogy. While other pedagogical schools of thought such as behaviourism are still valuable, the affordances of XR lend itself to learnercentred theory. Our review has shown that XR can operate within conventional pedagogical models such as constructivism. Based on the SOR model we have shown how XR can be used to enhance healthcare education, through a combination of interactivity, representational fidelity and immersion. However, further research into the predictive validity of XR's in healthcare education is required: does the learning translate into better performance and ultimately patient care? While it is beyond the scope of this paper to discuss this, there is also an increasingly important role for XR in a therapeutic context. ${ }^{60,61}$ However, it is paramount that the design and implementation of any new technology is driven by the pursuit of enhancing patient safety, experience, and care.

\section{Conclusion}

There is clear pedagogical basis for the use of XR in healthcare education, and its role is likely to evolve with changes in pedagogical theory. Translational research into learning outcomes is still in its infancy, but the results to date are extremely promising. $\mathrm{XR}$ implementation must focus on facilitating the interaction between students, teachers, and the learning environment. XR has the potential to cause a paradigm shift in healthcare education, but this is more likely to be successful if a learner-centred approach is adopted.

\section{Acknowledgements}

We thank Eric Topol for reviewing our manuscript.

\section{References}

1 Lee $\mathrm{H}-\mathrm{G}$, Chung S, Lee W-H. Presence in virtual golf simulators: The effects of presence on perceived enjoyment, perceived value, and behavioral intention. New Media Soc 2013;15:930-46.

2 Suh A, Prophet ]. The state of immersive technology research: a literature analysis. Comput Hum Behav 2018;86:77-90.

3 Unity. What is $A R, V R, M R, X R, 360$ ? https://unity3d.com/what-isxr-glossary.

4 Franklin Institute. What is extended reality $(X R)$ ? www.fi.edu/tech/ what-is-extended-reality (2020).

5 Marr B. What is extended reality technology? A simple explanation for anyone. Forbes, 12 August 2019. www.forbes.com/sites/ bernardmarr/2019/08/12/what-is-extended-reality-technologya-simple-explanation-for-anyone/.

6 Bowyer MW, Streete KA, Muniz GM, Liu AV. Immersive virtual environments for medical training. Semin Colon Rectal Surg 2008;19:90-7.

7 Haluck RS, Krummel TM. Computers and virtual reality for surgical education in the 21st century. Arch Surg 2000;135:786-92.

8 Mantovani F, Castelnuovo G, Gaggioli A, Riva G. Virtual reality training for health-care professionals. Cyberpsychol Behav 2003;6:389-95.

9 Ross BH. Psychology of learning and motivation: advances in research and theory. Elsevier, 2002.

10 Murphy P, Hall K, Soler J. Pedagogy and practice: culture and identities. SAGE, 2008.

11 UNESCO International Institute for Educational Planning. Effective and appropriate pedagogy. https://learningportal.iiep.unesco.org/ en/issue-briefs/improve-learning/teachers-and-pedagogy/ effective-and-appropriate-pedagogy.

12 Rost M. Generating student motivation. WorldView, 2006. www. pearsonlongman.com/ae/worldview/motivation.pdf.

13 Vavrus F, Thomas MAM, Bartlett L. Ensuring quality by attending to inquiry: learner-centred pedagogy in Sub-Saharan Africa. UNESCOIICBA, 2011.

14 Kompa JS. Disadvantages of teacher-centered learning. https:// joanakompa.com/2012/06/25/the-key-disadvantages-of-teachercentered-learning/.

15 Westbrook J, Durrani N, Brown R. Pedagogy, curriculum, teaching practices and teacher education in developing countries. Department for International Development, 2014. Available from www.gov.uk/government/publications/pedagogy-curriculumteaching-practices-and-teacher-education-in-developing-countries.

16 Ackermann E. Piaget's constructivism, Papert's constructionism: What's the difference? Future of Learning Group Publication 2001;5:438.

17 Goldie JGS. Connectivism: A knowledge learning theory for the digital age? Med Teach 2016;38:1064-9.

18 Dennick R. Constructivism: reflections on twenty five years teaching the constructivist approach in medical education. Int J Med Educ 2016;7:200-5.

19 Dewey ], Pate EG. Logic: the theory of inquiry. Henry Holt \& Co, 1938.

20 Wertsch JV. Vygotsky and the social formation of mind. Harvard University Press, 1985. Available from www.massey.ac.nz/ alock/ books/vigo-sfm.htm.

21 Perera NC. Constructivism, social constructivism and situated cognition: a sliding scale. Available from https://nishancperera. wordpress.com/2011/01/31/constructivism-social-constructivismand-situated-cognition-a-sliding-scale-by-nishan-perera/.

22 Murad M, Coto-Yglesias F, Varkey P, Prokop L, Murad L. The effectiveness of self-directed learning in health professions education: A systematic review. Med Educ 2010;44:1057-68.

23 Spencer JA, Jordan RK. Learner centred approaches in medical education. BMJ 1999;318:1280-3. 
24 Zhu E, Hadadgar A, Masiello I, Zary N. Augmented reality in healthcare education: an integrative review. Peer] 2014;2:e469.

25 Radianti J, Majchrzak TA, Fromm J, Wohlgenannt I. A systematic review of immersive virtual reality applications for higher education: Design elements, lessons learned, and research agenda. Comput Educ 2020;147:103778.

26 Bastian H, Glasziou P, Chalmers I. Seventy-five trials and eleven systematic reviews a day: how will we ever keep up? PLoS Med 2010;7:e1000326.

27 Baethge C, Goldbeck-Wood S, Mertens S. SANRA - a scale for the quality assessment of narrative review articles. Research Integr Peer Rev 2019;4:5.

28 Bschor T, Adli M. Treatment of depressive disorders. Dtsch Arzteblatt Int 2008;105:782-92.

29 Dede C. Immersive interfaces for engagement and learning. Science 2009;323:66-9.

30 Huang H-M, Rauch U, Liaw S-S. Investigating learners' attitudes toward virtual reality learning environments: Based on a constructivist approach. Comput Educ 2010;55:1171-82.

31 National Research Council. Virtual reality: scientific and technological challenges. National Academies Press, 1994.

32 Pan Z, Cheok AD, Yang H, Zhu J, Shi J. Virtual reality and mixed reality for virtual learning environments. Comput Graph 2006;30:20-8.

33 Dimitropoulos K, Manitsaris A, Mavridis I. Building virtual reality environments for distance education on the web: a case study in medical education. Int J Social Sci 2007;2:62-70.

34 Holmes J. Designing agents to support learning by explaining. Comput Educ 2007:48:523-47.

35 Brenton $\mathrm{H}$, Hernandez J, Bello F et al. Using multimedia and Web3D to enhance anatomy teaching. Comput Educ 2007;49:32-53.

36 Wankel C, Hinrichs, R. Engaging the avatar: New frontiers in immersive education. Information Age Publishing, 2012.

37 Zhang Y, Chen J, Miao D, Zhang C. Design and analysis of an interactive $\mathrm{MOOC}$ teaching system based on virtual reality. Int J Emerg Technol Learn 2018;13:111.

38 Kyaw BM, Saxena N, Posadzki P et al. Virtual reality for health professions education: systematic review and meta-analysis by the digital health education collaboration. J Med Internet Res 2019;21:e12959.

39 Jensen L, Konradsen F. A review of the use of virtual reality headmounted displays in education and training. Educ Inf Technol 2018;23:1515-29.

40 Norman G, Dore K, Grierson L. The minimal relationship between simulation fidelity and transfer of learning. Med Educ 2012;46:636-647.

41 Grady JL, Kehrer RG, Trusty CE et al. Learning nursing procedures: the influence of simulator fidelity and student gender on teaching effectiveness. J Nurs Educ 2008;47:403-8.

42 Munshi F, Lababidi H, Alyousef S. Low- versus high-fidelity simulations in teaching and assessing clinical skills. J Taibah Univ Med Sci 2015;10:12-15.

43 McMahan RP, Bowman DA, Zielinski DJ, Brady RB. Evaluating display fidelity and interaction fidelity in a virtual reality game. IEEE Trans Vis Comput Graph 2012;18:626-33.
44 Cummings J], Bailenson JN. How immersive is enough? A meta-analysis of the effect of immersive technology on user presence. Media Psychol 2016;19:272-309.

45 Slater M. Immersion and the illusion of presence in virtual reality. Br J Psychol 2018;109:431-3.

46 Dugdale M. Informed immersion. VRWorldTech Magazine, February 2020. https://issuu.com/vrworldtechmagazine/docs/vrwt_issue_2_ magazine/24.

47 Pottle J. Virtual reality and the transformation of medical education. Future Healthc ] 2019;6:181-5.

48 Gutierrez F, Pierce J, Vergara V et al. The effect of degree of immersion upon learning performance in virtual reality simulations for medical education. Stud Health Technol Inform 2007;125:155-60.

49 Makransky G, Terkildsen TS, Mayer RE. Adding immersive virtual reality to a science lab simulation causes more presence but less learning. Learn Instr 2019;60:225-36.

50 Mayer RE. Chapter Three - applying the science of learning to multimedia instruction. Psychology of Learning and Motivation, 2011;55:77-108.

51 Halarnkar $\mathrm{P}$, Shah S, Shah $\mathrm{H}$ et al. A review on virtual reality. Int J Computer Sci Issues 2012;9:325-30.

52 Sornette D, Davis AB, Ide K et al. Algorithm for model validation: Theory and applications. Proc Natl Acad Sci 2007;104:6562-7.

53 Frey B. The Sage encyclopedia of educational research, measurement, and evaluation (vols 1-4). Thousand Oaks, CA; Sage Publications, 2018.

54 Cronbach LJ, Meehl PE. Construct validity in psychological tests. Psychol Bull 1955;52:281-302.

55 Statistics How To. Construct validity: simple definition, statistics used. www.statisticshowto.datasciencecentral.com/construct-validity.

56 Barsom EZ, Graafland M, Schijven MP. Systematic review on the effectiveness of augmented reality applications in medical training. Surg Endosc 2016;30:4174-83.

57 Sugand K, Mawkin M, Gupte C. Validating Touch Surgery ${ }^{\mathrm{TM}}$ : A cognitive task simulation and rehearsal app for intramedullary femoral nailing. Injury 2015;46:2212-6.

58 Drew SA, Borsting E, Escobar AE et al. Can chronic visual discomfort measures accurately predict acute symptoms? Optom Vis Sci 2013;90:1149-55.

59 Knight JF, Baber C. Effect of head-mounted displays on posture. Hum Factors 2007:49:797-807.

60 da Silva Cameirão M, Bermúdez I Badia S, Duarte E, Verschure PFMJ. Virtual reality based rehabilitation speeds up functional recovery of the upper extremities after stroke: a randomized controlled pilot study in the acute phase of stroke using the rehabilitation gaming system. Restor Neurol Neurosci 2011;29:287-98.

61 Lange B, Flynn S, Proffitt R et al. Development of an interactive game-based rehabilitation tool for dynamic balance training. Top Stroke Rehabil 2010;17:345-52.

Address for correspondence: Dr Abison Logeswaran, Health Education England, Stewart House, 32 Russell Square, London, WC1B 5DN.

Email: abison.logeswaran.1@city.ac.uk 